\title{
Ultrasonography and computer tomography evaluation of hepatocellular carcinoma with cytohistopathological correlation
}

\author{
Hasnat Waheedul Hoque ${ }^{1}$, Shahinul Alam ${ }^{2}$, Sania Ahsan ${ }^{1}$ and Md. Nazrul Islam ${ }^{1}$ \\ ${ }^{1}$ Department of Radiology and Imaging; ${ }^{2}$ Department of Hepatology, Bangabandhu Sheikh Mujib \\ Medical University, Shahbag, Dhaka, Bangladesh.e-mail: wahaedh@gmail.com (HWH)
}

\begin{abstract}
The prevalence of hepatocellular carcinoma (HCC) in Bangladesh is 35\% among all liver diseases. Sonographic examinations were performed for the evaluation of 38 cases of HCC; then, CT examinations were done of these cases subsequently. Expert opinion was taken in each case for both modalities. Age, sex, clinical features, location of the hepatic lesion, multiplicity, echo-character, CT density, and, contrast enhancement were evaluated in all cases. Histocytopathology reports were collected from the patients and were correlated with the ultrasonography and CT findings. Thirty five cases were detected as HCC on ultrasonography and 36 cases in CT scan. In ultrasonography, most of the lesions $(82.9 \%)$ were found in right lobe, maximum lesions $(45.7 \%)$ were hypoechoic and lesion showed mosaic pattern in $68.6 \%$ cases, lateral shadowing in $34.3 \%$ and posterior acoustic enhancement in $45.7 \%$ cases. Significant difference found between mosaic pattern and lateral shadowing $(\mathrm{p}<0.05)$. On CT scan, majority of lesions (50\%) were hypodense, $91.7 \%$ lesions were contrast enhanced. Pattern of enhancement was mostly heterogeneous. Both of the modalities found sensitive but CT was found more sensitive, specific and accurate than ultrasonography in detecting HCC.
\end{abstract}

\section{Introduction}

Hepatocellular carcinoma (HCC) causing an estimated 1 million deaths in the world per year. Its incidence in developing country is high. It is the third most malignancy among all types of cancers. Epidemiological study shows that HCC is very common in Asia and Sub-Saharan Africa and uncommon in developed countries ${ }^{1,2}$. In developing countries, incidence rate is two to three-fold higher than in developed countries. Excess liver cancer incidence among men compare to women is universal $^{2}$. It is also found that $\mathrm{HCC}$ has an increasing incidence during the last century ${ }^{3,4}$. In Bangladesh its prevalence is 35\% among all liver diseases 5 . Study shows HBV contribution $47 \%$ in causation of $\mathrm{HCC}^{5}$ and $\mathrm{HCV}$ accounts for $56 \%{ }^{6}$. Cirrhosis of liver also is a major (18.75\%) contributor to HCC in Bangladeshi population ${ }^{5}$. This is similar to other studies in Himalayan subcontinent $^{7-10}$.

Significant advances in cross sectional imaging modalities like ultrasonography and CT now allow not only detection but often non-invasive charac- terization of focal and diffuse hepatic processes. Several studies were undertaken regarding the sonographic or CT evaluation of HCC. The sensitivity of real time sonography for detection of small HCCs has been established ${ }^{11,12}$.

Ultrasonography had the highest detection rate $(91.2 \%)$ for $\mathrm{HCC}<3 \mathrm{~cm}$ in diameter compare to $\mathrm{CT}$ $(63.2 \%)$. For HCC $3-5 \mathrm{~cm}$ in diameter, the difference in detection rate becomes smaller which is $92.9 \%$ for ultrasonography and $81.8 \%$ for $\mathrm{CT}^{13}$.

For $\mathrm{HCC} \leq 2 \mathrm{~cm}$ in diameter delectability rate for ultrasonography is $52 \%$ and that of contrast CT is $56 \%$. For $\mathrm{HCC} \leq 3 \mathrm{~cm}$ in diameter delectability rate for ultrasonography is $85 \%$ and that of contrast CT is $100 \%{ }^{14}$.

As surgical resection is the most effective treatment of HCC, it needs early detection. Prognosis of HCC is extremely poor because of the difficulty in early detection. But small HCC discovered by a mass screening program have a very good prognosis when successfully resected. Therefore periodical follow up of high risk patients by imaging 
techniques is essential for the early detection of HCCs.

Among the modern imaging modalities, ultrasonography and CT scan was used to detect and evaluate HCCs. This study also evaluate whether ultrasonography can effectively used, in detection and follow up of case of HCC, as screening modality.

\section{Materials and Methods}

This prospective study was carried out in the Department of Radiology and Imaging, BSMMU, Dhaka from July 2004 to June 2005 based on an ethically cleared approved protocol. Thirty eight patients having complaints suggestive of HCC from 24 years to 70 years of age of either sex with raised serum alpha-fetoprotein beyond $20 \mathrm{ng} / \mathrm{ml}$ were included in this study. All patients were informed about the nature, objectives and procedure of the study in understandable language. Written informed consent was taken with the assurance of keeping the personal information concealed. A pre-tested questionnaire was used for data collection. Patients were evaluated by detailed history, clinical examination with emphasis on hepatobiliary system. Sonographic examination was performed for the evaluation of HCC. Then CT scan was done of the same patient, subsequently. Expert opinion was taken in each case for both the modalities.

Ultrasonographic technique: Every patient had been fasting for 8-10 hours previous to sonographic examination. Ultracarbon tablets were given for minimizing abdominal free gas. Ultrasonography intervention was performed by Sonoline Adora machine (SEIMENS) with $3.5 \mathrm{MHz}$ curvilinear transducer. Scanning was performed with the patient in a supine, right and/left anterior oblique and/ right lateral decubitus position. Patient was asked to hold his/her breath at different phase of respiration and sagittal, transverse and oblique scans were done through sub costal, and intercostal spaces, in a symmetrical fashion to screen the whole liver. Number, site, nature, echogenicity, lobar distribution of the lesion were recorded.

CT technique: CT scan was done after US detection of the HCC. Scan was done by third generation helical CT machine (Hitachi/W2000). CT obtained in cranio-caudal direction with the following parameters- 165 effective $\mathrm{mA}, 120 \mathrm{KVp}, 1: 1.5$ pitch, collimation- $5 \mathrm{~mm}$, effective section thickness 3-5 $\mathrm{mm}$ at 5-10 $\mathrm{mm}$ interval. IV injection was given by $18 \mathrm{G}$ needle in ante-cubital vein. Contrast was used Iopamiro $60 \mathrm{ml}$. CT was interpreted by the researchers' panel and reviewed by professors of the department. Number, nature, density (after and before contrast), and lobar distribution of the lesion was recorded.

Cytopathology technique: Ultrasonography guided aspiration from the lesion was done by $18 \mathrm{G}$ needle under full aseptic precaution. Then the aspirate spread in a glass slide and fixed in absolute alcohol. Slides then sent for laboratory examination. The Papaniculaus' stain is done and examined under microscope.

Age, sex, clinical features, location of the lesion, multiplicity, echo character/CT density and contrast enhancement were evaluated in all cases. Data were tabulated and analyzed by SPSS by using computer. Cyto-histopathology reports were correlated with the ultrasonography/CT findings. Sensitivity, specificity, accuracy, along with positive/negative predictive values of both modalities was calculated by standard statistical formulae.

\section{Results}

Among 38 patients, the mean $( \pm \mathrm{SD})$ age was 50 $( \pm 13.69)$ years. No significant age group preponderance was noted for HCC incidence. There were 32 males and 6 females. Male female ratio was 5.3:1. In this study, highest number of HCC patients $(34.2 \%)$ was small scale businessman. Cultivator (23.7\%), housewife (15.8\%), serviceman $(10.5 \%)$ and miscellaneous $(15.8 \%)$ profession were also included in this study. No significance preponderance between different occupation groups was noted. Among studied patients, most frequent symptom was upper abdominal pain (73.7\%) followed by (right) abdominal mass (57.9\%). Ascites (31.6\%), anorexia (26.3\%), weight loss $(23.6 \%)$ and fever $(21.2 \%)$ were also found.

Among 38 cases, 35 cases were detected by ultrasonography as HCC. Most of the patients got right lobe lesions $(82.9 \%)$. Lesions in left lobe were in $11.4 \%$ and in both lobes in $5.7 \%$ cases (Table I).

Table I: Location and numeral of HCC

\begin{tabular}{lcc}
\hline & $\begin{array}{c}\text { Ultrasonography } \\
(\mathrm{n}=35)\end{array}$ & $\begin{array}{c}\mathrm{CT} \\
(\mathrm{n}=36)\end{array}$ \\
\hline Location & 29 & \\
Right Lobe & 4 & 29 \\
Left Lobe & 2 & 4 \\
Both Lobes & & 3 \\
Numeral & 25 & 25 \\
Unifocal & 10 & 11 \\
Multifocal & & \\
\hline
\end{tabular}


Maximum lesions (71.4\%) were found unifocal; 10 $(28 \%)$ lesions were multifocal.

Hypoechoic lesions (45.7\%) top the table. In 13 patients $(37.1 \%)$ lesion showed mixed echogenicity and in $6(17.2 \%)$ patients' lesions were hyperechoic (Table II). Mosaic pattern and lateral shadowing was characteristic in $24(68.6 \%)$ and $12(34.3 \%)$ cases respectively; whereas $16 \quad(45.7 \%)$ cases showed posterior acoustic enhancement. Significant difference is noted between mosaic pattern and lateral shadowing $(\mathrm{z}=2.1 ; \mathrm{p}<0.05)$.

In CT evaluation, 36 cases were detected as HCC. Twenty nine $(80.6 \%)$ patients got lesions in right lobe. Lesions in left lobe were found in 4 patients. Lesions in both lobes of liver were found in 3 cases. Maximum (25 cases) lesions were observed unifocal where as multifocal lesions were detected in 11 patients. Eighteen patients got hypodense lesion (Table II). Ten lesions showed mixed density. After injection of contrast agent 33 $(91.7 \%)$ lesions were enhanced, which was mostly heterogenous. Three $(8.3 \%)$ lesions were unenhanced.

Comparing both the modalities in detecting HCC with cyto-histopathological reports (Table III), it was found that, sensitivity, specificity, accuracy, positive predictive value and negative predictive value for ultrasonography were $94.5 \%, 100 \%$, $94.7 \%, 100 \%$ and $33.3 \%$ respectively. Sensitivity, specificity, accuracy, positive predictive value and negative predictive value for CT were $97.3 \%$, $100 \%, 97.4 \%, 100 \%$ and $50 \%$ respectively.

Table II: Distribution of HCC patients by pattern of lesions

\begin{tabular}{lcc}
\hline $\begin{array}{l}\text { Echogenicity/ } \\
\text { Density }\end{array}$ & $\begin{array}{r}\text { Ultrasonography } \\
(\mathrm{n}=35)\end{array}$ & $\begin{array}{c}\mathrm{CT} \\
(\mathrm{n}=36)\end{array}$ \\
\hline Hypo & 16 & 18 \\
Iso & 0 & 2 \\
Hyper & 6 & 6 \\
Mixed & 13 & 10 \\
\hline
\end{tabular}

Table III: Comparison of ultrasonography and CT with histocytopathological findings

\begin{tabular}{llcc}
\hline & & \multicolumn{2}{c}{ Histocytopathology } \\
\cline { 3 - 4 } & & Positive & Negative \\
\hline Ultrasonography & Positive & 35 & 0 \\
& Negative & 2 & 1 \\
CT & Positive & 36 & 0 \\
& Negative & 1 & 1 \\
\hline
\end{tabular}

\section{Discussion}

The purpose of the study was the determine accuracy of ultrasonography and CT in detection and evaluation of HCC. As the patients of HCC have rapidly deterioration courses, early detection is essential for surgical treatment ${ }^{15}$. Early detection of HCC has improved with non invasive imaging modalities, such as ultrasonography, CT and magnetic resonance imaging.

Ultrasonography is one of the useful tool for detecting focal liver diseases and its advantages over other modalities in early detection and characterization of HCC. It is safe, cheap, easily available, can be done rapidly and repeatedly without any radiation hazards. Real time imaging is also possible.

CT is quite valuable in diagnosing and evaluating HCC. However, the presence of isodense tumor should always by kept in mind false positive examination may result from very small lesion, low contrast between tumor and surroundings liver tissue or due to technical failure. In this study there are two false negative in ultrasonography and one in CT. With the spiral CT diagnostic decision tree for the management of $\mathrm{HCC}$ has dramatically changed $^{16}$. Ultrasonography, CT, magnetic resonance imaging techniques seem to have a strong potential to improve detection and characterization techniques seen to have a strong potential to improve detection and characterization of $\mathrm{HCC}^{30}$. CT has been highly useful in detecting liver tumors and determining their extent ${ }^{17}$.

In this study, out of 38 cases 35 were diagnosed as HCC by ultrasonography and 36 cases were diagnosed by CT. Right lobe of the liver alone involved in $82.9 \%$ cases in ultrasonography and $80.5 \%$ cases in CT. In ultrasonography $11.4 \%$ lesions detected in left lobe, which was $11.1 \%$ in CT. Ultrasonography detects $5.7 \%$ lesions in both lobes whereas with CT $8.3 \%$ cases are detected.

Saad et al 1996 found 59\% right lobe lesion and $23 \%$ left lobe lesion. This study is not similar to our findings, probably because of variation in patient selection ${ }^{18}$.

In ultrasonography $71.4 \%$ cases found as unifocal lesion, where as in CT $69.4 \%$ lesions were found unifocal. Ultrasonography detected $28.6 \%$ multifocal lesions; CT showed $30.6 \%$ lesions as multifocal. Yoshida et al 1987 in an ultrasonography study found $68.8 \%$ as unifocal lesion ${ }^{19}$. Sarder et al 1997 found $71.4 \%$ unifocal and $27.6 \%$ multifocal lesions ${ }^{20}$. Colombo et al., 1991 found $71 \%$ HCC lesion as unifocal in sonography and 
$28 \%$ as multifocal ${ }^{21}$. Our study is more or less similar with these studies.

Types and patterns of echogenicity is an important characteristic of tumor, which helps in detection and differentiations of HCC from other hepatic masses. Tanaka et al., 1993 studied 23 patients of HCC, sonographically. They found $26.1 \%$ hypoechoic, $60.8 \%$ complex or mixed and $13.04 \%$ hyperechoic $^{22}$. Giorgi et al., 2004 found $60.8 \%$ hypoechoic tumors in sonographic scan of HCC patients $^{23}$. Tanaka et al., 1993 found HCC lesion hypoechoic in less percent than our study but Giorgi et al., 2004 found more that us. This may be due to lesion size.

In our study $50 \%$ lesion were hypodense, $27.7 \%$ were mixed, $5.6 \%$ were isodense, and $16.7 \%$ were hyperdense. Total $91.7 \%$ cases were enhanced after contrast injection.

In a CT study with 13 HCC patients by Saad et al., 1996, 76\% lesions were hypodense, 7.6\% were hyperdense $^{18}$. In another CT study with $51 \mathrm{HCC}$ patients. Valls et al., 2003 found $88 \%$ of $\mathrm{HCC}$ nodules enhanced at arterial phase ${ }^{24}$. Our study findings were close to the findings of other researchers.

An ultrasonography study of $\mathrm{HCC} 64$ patients Yoshida et al., 1987 found mosaic pattern in $65.6 \%$, posterior echo-enhancement in $53.1 \%$ and lateral shadowing in $32.8 \%{ }^{19}$. In our study, mosaic pattern is found in $68.6 \%$ cases, posterior echoenhancement in $45.7 \%$ cases and lateral shadowing in $34.3 \%$. Our study was in alignment with Yoshida et al.

In our study, sensitivity, specificity, accuracy, PPV and NPV for ultrasonography were 94.5\%, 100\%, $94.7 \%, 100 \%$ and $33.3 \%$ respectively. Sensitivity, specificity, accuracy, PPV and NPV for CT was $97.3 \%, 100 \%, 97.4 \%, 100 \%$ and $50 \%$ respectively.

Sinagawa et al., 1984 found overall Ultrasonography sensitivity in detecting $\mathrm{HCC}$ is $92.2 \%$ which is closely similar to our findings. They found CT sensitivity $73.2 \%^{13}$. But Giorgio et al., 2003 found CECT sensitivity $91.9 \%{ }^{23}$. Snow et al., 1979 found CT sensitivity $96 \%$, specificity $86 \%$, accuracy $91 \%$ and ultrasonography sensitivity $75 \%$, specificity $50 \%$, and accuracy $90 \%{ }^{17}$.

Ultrasonography and CT have been used as modality for screening and evaluation. In regular survillance program it is found that, $75 \%$ to $90 \%$ of HCCs occur as a single lesion less than $5 \mathrm{~cm}$. The sensitivity of ultrasonography for detecting small HCC ranges from $55 \%-85 \%$ and specificity is between $90 \%-94 \%$. The sensitivity and specificity of $\mathrm{CT}$ in detecting $\mathrm{HCC}$ varies from size to size of the lesion. Sensitivity of CT is greater than $90 \%$ for tumors greater than $1 \mathrm{~cm}$ in size. In general, sensitivity of contrast enhanced $\mathrm{CT}$ ranges from $53 \%$ to $95 \%$. CT scan specificity in detecting HCC is approximately $94 \%{ }^{25}$. Sensitivity, specificity, accuracy for CT and ultrasonography found in our study were in alignment with other studies.

$\mathrm{CT}$ is found more sensitive and accurate. But ultrasonography is the modality which is easily available in most areas of the country. As a screening modality ultrasonography may be used but for proper characterization of the hepatic lesion CT is necessary.

\section{Acknowledgement}

We are grateful to Bangladesh Medical Research Council (BMRC) for partial funding to this research work.

\section{Authors' contribution}

HWH: Study planning, design, data collection, compiling, analysis and manuscript writing.

ShA: Study planning, design, data collection and analysis.

SA: Study planning, design and data collection.

MNI: Study planning, design, and data collection.

\section{References}

1. Beasley RP, Hwang LY, Lin CC, Chien CS. Hepatocellular carcinoma and Hepatitis B virus: A prospective study of 22,707 cases men in Taiwan. Lancet 1981; 2: 1129-33.

2. Bosch FX, Ribes J, Borras J. Epidemiology of primary liver cancer. Semin Liv Dis. 1999; 19: 271-83.

3. Beasley RP, Hwang LY. Overview of the epidemiology of hepatocellular carcinoma. In: Viral hepatitis and liver diseases. Hollinger FB, Lemon SM, Margolis H (eds). New York, Williams and Wikins, 1991, pp 532-35.

4. Sinnatamby CS. Last's Anatomy- Regional and applied. $10^{\text {th }}$ ed. Edinburgh, Churchill Livingstone, 1999, pp 253-57.

5. Khan M, Haq SA, Ahmad N, Matin MA. Etiology and clinical profile of hepatocellular carcinoma in Bangladesh. Bangladesh Med Res Counc Bull. 1997; 23: 16-24.

6. Zaman S, Khan M, Alam K, Williams R. Primary hepatocellular carcinoma and viral hepatitis B and C infection in Bangladeshi subjects. J Trop Med Hyg. 1995; 98: 64-68.

7. Shresta SM. Primary hepatocellular carcinoma in Nepal: Report on hepatocellular carcinoma in Asia. Kobe International Centre for Medical Research, Kobe University School of Medicine, 1985, pp 47-51.

8. Joshi A, Ayesha Q, Habibullah CM. Immunological studies in HBV related chronic liver disease. J Pathol Microl. 1990; 3: 351-54. 
9. Qureshi H, Zuberi SJ, Jafarey NA, Zaidi SHN. Liver and biliary hepatocellular carcinoma in Karachi. Gastroenterol Hepatol. 1990; 5: 1-6.

10. Sebastien M, Ichpujaini RI, Kumari S. Incidence of different type of viral hepatitis in Delhi, Uttar Pradesh and Rajasthan. J Com Dis. 1990; 22: 129-33.

11. Sheu JC, Chen DS, Sung IL, Chuang CN, Yang PM, Lin JT, Yang PC. Hepatocellular carcinoma: US evaluation in the early stage. Radiology 1985; 155 : 463-67.

12. Kudo M, Hirasa M, Takakuwa H, Ibuki Y, Fujimi K, Miyamura M, Tomita S, Komori H, Todo A, Kitaura

Y. Small hepatocellular carcinoma in chronic liver disease: Detection with SPECT. Radiology 1986; 159: 697-703.

13. Shinagawa T, Ohto M, Kimura K, Tsunetomi S, Morita M, Saisho H, Tsuchiya Y, Saotome N, Karasawa E, Miki M, Ueno T, Okuda K. Diagnosis and clinical features of small hepatocellular carcinoma with emphasis on utility of real-time ultrasonography. Gastroenterology 1984; 86: 495-502.

14. Choi BI, Kim CW, Han MC, Kim CY, Lee HS, Kim ST, Kim YI. Sonographic characteristics of small hepatocellular carcinoma. Gasterointest Radiol. 1989; 14: 255-61.

15. Choi BI. The current status of imaging diagnosis of hepatocellular carcinoma. Liver Transplantation 2004; 10 (2 Suppl): 20-25.

16. Kudo M. Imaging diagnosis of hepatocellular carcinoma and premalignant/borderline lesions. Semin Liv Dis. 1999; 19: 297-309.

17. Snow JH Jr, Goldstein HM, Wallace S. Comparison of scintiography, sonography, and computed tomography in the evaluation of hepatic neoplasm. AJR 1979; 132: 915-18.
18. Saad AMH, Alam SZ, Alam J. Evaluation of liver mass by ultrasound, CT scan and FANC- A study of 22 cases. BJRI. 1996; 4: 7-10.

19. Yoshida T, Matsu H, Okazaki N, Yoshino M. Ultrasonographic differentiation of hepatocellular carcinoma from metastatic liver cancer. J Clin Ultrasound 1987; 15: 431-37.

20. Sardar MK, Shamsuddin AKM, Kundu SS. Hepatocellular carcinoma and metastatic liver cancer: ultrasonographic differentiation- Study of 19 cases. BJRI. 1997; 5: 26-29.

21. Colombo M. Hepatitis C virus and hepatocellular carcinoma. Semin Liv Dis. 1999; 19: 263-69.

22. Tanaka S, Kitamura T, Imaoka S, Sasaki Y, Taniguchi $\mathrm{H}$, Ishiguro S. Hepatocellular carcinoma: Sonographic and histologic correlation. Am J Roentgenol. 1983; 140: 701-07.

23. Giorgi A, Ferraioli G, tarantio L, de stafano G, Scala V, Scarano F, Coppola C, Viscovo LD. Contrast enhanced sonographic appearance of hepatocellular carcinoma in patients with cirrhosis: comparison with contrast enhanced helical CT appearance. AJR. 2004; 183: $1319-26$.

24. Valls C, Mònica Cos , Figueras J, Andía E, Ramos E, Sánchez A, Serrano T, Torras J. Pre-transplantation diagnosis and staging of hepatocellular carcinoma in patients with cirrhosis: value of dual-phase helical CT. AJR. 2004; 182: 1011-17.

25. Saab S, Ly D, Nieto J, Kanwal F, Lu D, Raman S, Amado R, Nuesse B, Durazo F, Han S, Farmer DG, Ghobrial RM, Yersiz H, Chen P, Schwegel K, Goldstein LI, Tong M, Busuttil RW. Hepatocellular carcinoma in patients waiting for liver transplantation: a decision analytic model. Liver Transplantation 2003; 9: $672-81$. 